\title{
Análise comparativa de técnicas de escaneamento digital: estudo in vitro
}

Análisis comparativo de las técnicas de escaneo digital: estudio in vitro

Comparative analysis of digital scanning techniques: in vitro study

\section{Eduarda Bianchini CECHELERO}

Mariá Cortina BELLAN ${ }^{2}$

Maurício André $\mathbf{B I S I}^{\mathbf{3}}$

${ }^{1}$ Curso de Graduação em Odontologia, Centro Universitário da Serra Gaúcha - FSG 95020-472 Caxias do Sul - RS, Brasil

${ }^{2}$ Mestre em Prótese Dentária, Professora do Curso de Odontologia, Centro Universitário da Serra Gaúcha - FSG 95020-472 Caxias do Sul - RS, Brasil
${ }^{3}$ Doutor em Prótese Dentária, Professor do Curso de Odontologia, Centro Universitário da Serra Gaúcha - FSG 95020-472 Caxias do Sul - RS, Brasil

\section{Resumo}

Objetivos: O presente estudo tem como objetivo avaliar e comparar a técnica recomendada pelo fabricante e uma técnica de variação padrão de dois scanners intraorais em relação a um escaneamento com um scanner de mesa e analisar a possibilidade de alteração dimensional. Materiais e Métodos: Foram confeccionados 5 grupos $(n=5)$, sendo o $G=1$ com AutoScan-DS-EX sendo fluxo de trabalho automático, $G=2$ com TRIOS 3 e técnica recomendada pelo fabricante, $G=3$ com TRIOS 3 e técnica modificada, $G=4$ com iTero e técnica recomendada pelo fabricante e $G=5$ com iTero e técnica modificada, e foram submetidos a uma análise qualitativa de cor pelo software CloudCompare. Resultados: Foi presente bastante alteração dimensional entre os Grupos 2 e 3, 4 e 5, pouca alteração dimensional entre os Grupos 1 e 3, 1 e 5, e nenhuma alteração dimensional entre os Grupos 1 e 2, e 1 e 4. Conclusão: Conclui-se que a modificação da técnica recomendada pelo fabricante altera o padrão final do modelo digitalizado principalmente entre os scanners intraorais, sendo que houve pouca alteração quando as técnicas foram comparadas com um scanner de bancada. A qualidade dos escaneamentos se baseia na quantidade de pontos coletados apresentando-se maior nos escaneamentos com scanner de mesa.

Descritores: Técnica de Moldagem Odontológica; Modelos Dentários; Imageamento Tridimensional.

\section{Abstract}

Objectives: This study aims to evaluate and compare the technique recommended by the manufacturer and a standard variation technique of two intraoral scanners in relation to a flatbed scanner and to analyze the possibility of dimensional change. Materials and Methods: 5 groups were made $(n=5), G=1$ with AutoScan-DS-EX being automatic workflow, $G=2$ with TRIOS 3 and manufacturer recommended technique, $\mathrm{G}=3$ with TRIOS 3 and modified technique, $\mathrm{G}=4$ with iTero and technique recommended by the manufacturer and $G=5$ with iTero and modified technique, and were subjected to a qualitative color analysis by CloudCompare software. Results: There was a large dimensional change between Groups 2 and 3, 4 and 5, little dimensional change between Groups 1 and 3, 1 and 5, and no dimensional change between Groups 1 and 2 , and 1 and 4 . Conclusion: It is concluded that the modification of the technique recommended by the manufacturer alters the final pattern of the digitized model mainly among intraoral scanners, and there was little change when the techniques were compared with a flatbed scanner. The quality of scans is based on the number of points collected and higher on flatbed scans.

Descriptors: Dental Impression Technique; Dental Models; Imaging, Three-Dimensional.

\section{Resumen}

Objetivos: Este estudio tiene como objetivo evaluar y comparar la técnica recomendada por el fabricante y una técnica de variación estándar de dos escáneres intraorales en relación con un escáner de superficie plana y analizar la posibilidad de cambio dimensional. Materiales y métodos: Se formaron 5 grupos $(n=5), G=1$ con AutoScan-DS-EX como flujo de trabajo automático, $\mathrm{G}=2$ con TRIOS 3 y técnica recomendada por el fabricante, $\mathrm{G}=3$ con TRIOS 3 y técnica modificada, $\mathrm{G}=4$ con iTero y la técnica recomendada por el fabricante y $G=5$ con iTero y la técnica modificada, y fueron sometidos a un análisis de color cualitativo por el software CloudCompare. Resultados: Hubo un gran cambio dimensional entre los Grupos 2 y 3,4 y 5 , un pequeño cambio dimensional entre los Grupos 1 y 3, 1 y 5, y ningún cambio dimensional entre los Grupos 1 y 2 , y 1 y 4 . Conclusión: Se concluye que la modificación de la técnica recomendada por el fabricante altera el patrón final del modelo digitalizado principalmente entre los escáneres intraorales, y hubo pocos cambios cuando las técnicas se compararon con un escáner de superficie plana. La calidad de los escaneos se basa en el número de puntos recogidos y es mayor en los escaneos de superficie plana.

Descriptores: Técnica de Impresión Dental; Modelos Dentales; Imagenología Tridimensional.

INTRODUÇÃO

$\mathrm{Na}$ Odontologia o planejamento consiste não somente na avaliação clínica e radiográfica, mas também na obtenção de modelos de estudo para análise de casos. Atualmente para se obter uma cópia com precisão da cavidade oral podemos destacar os escaneamentos intraorais ${ }^{1}$. Com esta tecnologia é possível realizar diversos procedimentos clínicos e laboratoriais como diagnóstico e plano de tratamento para cirurgias ortognáticas, guias cirúrgicas para implantes, reabilitação protética e planejamento ortodôntico ${ }^{2,3}$.

O escaneamento digital pode ser obtido por diferentes técnicas, intraoral e extraoral, sendo que os sistemas atuais utilizam múltiplas varreduras e as imagens obtidas são cruzadas em áreas sobrepostas para obter as estruturas virtuais bem definidas. Cada sistema de scanner tem um modo de captura de dados de acordo com 0 princípio de captura de imagem $^{3}$. Sobretudo, a técnica de escaneamento, a habilidade e o conhecimento do profissional são imprescindíveis para 0 sucesso da reprodutibilidade e confiabilidade do escaneamento, independente da forma que é obtida, intraoral ou extraoral.

Contudo, a tecnologia digital de escaneamento tem suas limitações e uma análise minuciosa sobre a possibilidade de alteração dimensional dos modelos digitais deve ser considerada para promover uma moldagem digital eficiente proporcionando uma reabilitação oral adequada.

Portanto o objetivo desse estudo é 
avaliar se diferentes estratégias de varredura podem promover alteração dimensional na digitalização de um arco completo e comparar a técnica recomendada pelo fabricante e uma técnica de variação padrão de dois scanners intraorais em relação a um escaneamento com um scanner de mesa.

MATERIAL E MÉTODO

O presente estudo foi uma análise experimental in vitro de diferentes estratégias de varredura de dois scanners intraorais comparados a um scanner de mesa de uma arcada superior completa, sendo o local de experimento do estudo o Complexo Odontológico do Centro Universitário da Serra Gaúcha - FSG, em Caxias do Sul - RS.

As estratégias de varredura foram realizadas sobre um modelo de gesso confeccionados sobre um Manequim de Dentística Pré-fabricado de Acrílico (PRONEW TOP PD100, Rio de Janeiro, BR) com 16 dentes hígidos. Foram avaliados no estudo três sistemas de impressão digital: TRIOS (3shape, Warren, NJ), iTero element (Align Technology, San Jose, CA) e AutoScan-DS-EX (Shining 3D, Hangzhou, China).

Primeiramente foi realizada uma moldagem com Silicone de Adição (Express XT, 3M ESPE), foi vertido após 2 horas da moldagem o Gesso Pedra tipo IV (Fujirock Amarelo Pastel, GC América) com tempo de presa de 12 minutos e armazenado em local seco e limpo. As varreduras experimentais foram feitas por um único operador experiente com cada um dos três sistemas digitais de impressão, de cada um dos testes padrões da varredura. Os padrões de digitalização selecionados são os recomendados pelos fabricantes para cada sistema de impressão digital e um padrão modificado. Cada padrão de digitalização produziu um arquivo de estereolitografia em formato STL (Standard Triangle Language) mestre que, quando importado para um software de análise tridimensional (3D) CloudCompare V2 (Telecom ParisTech e EDF, 2003) permitiu o alinhamento de arquivos STL pela sobreposição das malhas.

A técnica de varredura recomendada pelos fabricantes do scanner TRIOS tem início pela oclusal do segundo molar direito, em movimento para o lado esquerdo do arco, retornando do lado palatino dos dentes até o lado direito novamente e por último voltando pela superfície vestibular até o lado esquerdo. $E$ para o scanner iTero elemento possui a mesma sequência, porém fazendo uma varredura final iniciando pela palatina dos dentes anteriores e finalizando na vestibular. $O$ padrão modificado de varredura que o estudo sugere deve iniciar pelo segundo molar direto fazendo uma varredura em onda, voltando pela palatina até o lado direito e finalizando pela vestibular até o lado esquerdo.

A quantidade de grupos selecionados para esse estudo é proporcional à quantidade de estratégias de varredura a serem analisadas, ou seja, 5 grupos com 1 amostra cada $(n=5)$ :

-Grupo 1: Grupo controle, na qual foi realizada varredura com o scanner de mesa AutoScan-DS-EX tendo fluxo de trabalho automático.

-Grupo 2: TRIOS com estratégia de varredura recomendada pelo fabricante.

-Grupo 3: TRIOS com estratégia de varredura de um padrão modificado.

-Grupo 4: iTero element com estratégia de varredura recomendada pelo fabricante.

-Grupo 5: iTero element com estratégia de varredura de um padrão modificado.

O modelo foi avaliado após ser submetido as varreduras e importado ao software CloudCompare, na qual gerou um gráfico de cores e medidas das quantidades de malhas em relação as distorções apresentadas. O grupo 1 (grupo controle) foi comparado aos grupos 2,3,4 e 5. E depois os grupos da mesma marca de scanner foram comparados entre si, grupo 2 por 3 , e grupo 4 por 5 . As comparações foram classificadas de acordo com a cor apresentada nos gráficos e classificada em: nenhuma, moderada ou bastante alteração dimensional. Nenhuma alteração dimensional (somente áreas verdes), moderada alteração dimensional (verde escuro, verde e amarelo) ou bastante alteração dimensional (azul, verde, amarelo e vermelho). Além da análise da coloração foi avaliado a qualidade dos escaneamentos de acordo com a quantidade de pontos captados pelo software.

RESULTADOS

Na primeira análise quando comparamos o Grupo 1 ao Grupo 2 (Figura 1) nos mostra que não houve nenhuma alteração dimensional entre as duas varreduras, sem apresentar diferenças entre as áreas sobrepostas (Figura 2 e 3). Nessa análise a quantidade foi de 229607 pontos captados.

$\mathrm{Na}$ segunda análise (Figura 4) comparamos o Grupo 1 ao Grupo 3 e pode ser classificado com moderado grau de alteração dimensional, tendo as áreas de maior distorção os dentes posteriores (Figura 5 e 6). $\mathrm{Na}$ segunda análise a quantidade de pontos foi de 221696. 


\section{Quando comparados os Grupos $1 \mathrm{e}$} Grupo 4 na terceira análise (Figura 7), observamos que não houve nenhuma alteração dimensional, não apresentando diferenças entre as sobreposições das áreas (Figura 8 e 9). Nessa análise a quantidade de pontos captados foi de 217394.

$\mathrm{Na}$ comparação da análise quatro (Figura 10) os Grupos 1 e Grupo 5 apresentaram resultados de moderada alteração dimensional tendo como áreas de maior distorção os dentes posteriores na região de terceiro molar (Figura 11 e 12). Na quarta análise a quantidade foi de 213824 pontos.

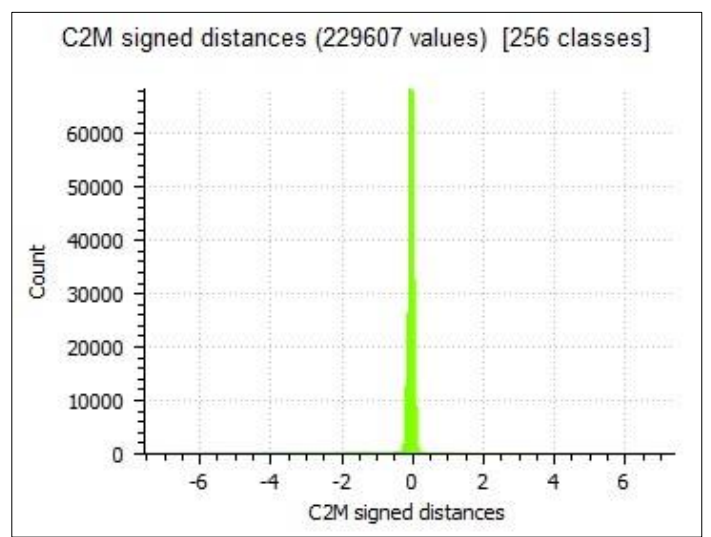

Figura 1: Variação de cor dos grupos 1 e 2 em relação as suas superficies totais comparadas e as distâncias das malhas dos stl.

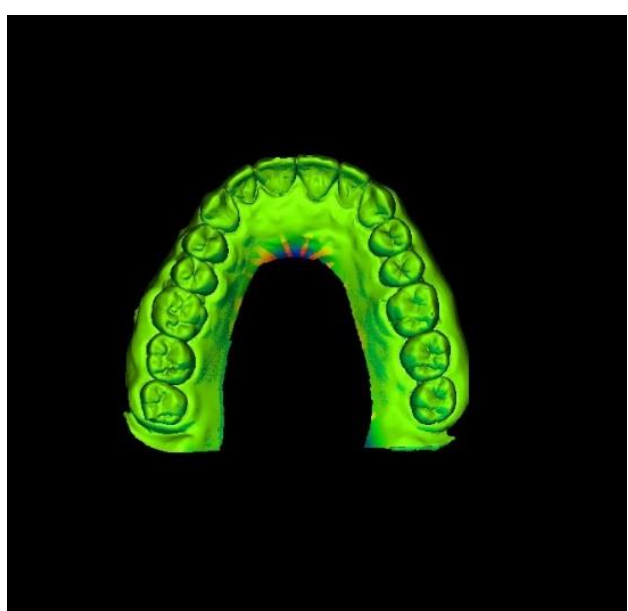

Figura 2: stl. comparativo do grupo 1 e grupo 2 com vista oclusal

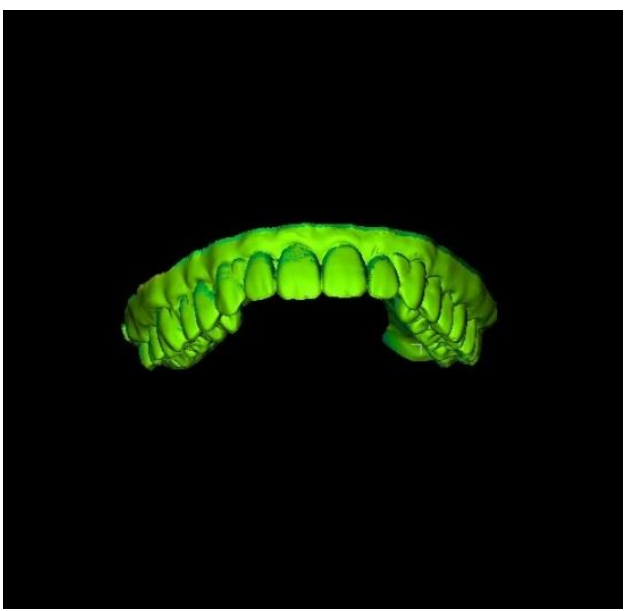

Figura 3: stl. comparativo do grupo 1 e grupo 2 com vista frontal

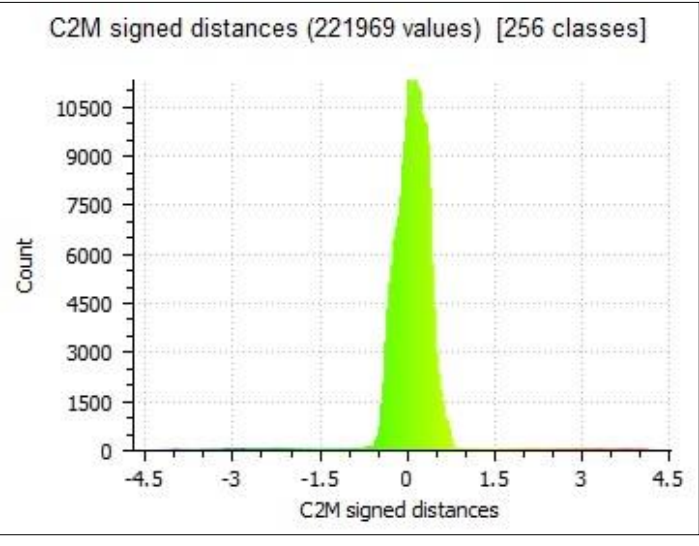

Figura 4: Variação de cor dos grupos 1 e 3 em relação as suas superficies totais comparadas e as distâncias das malhas dos stl.

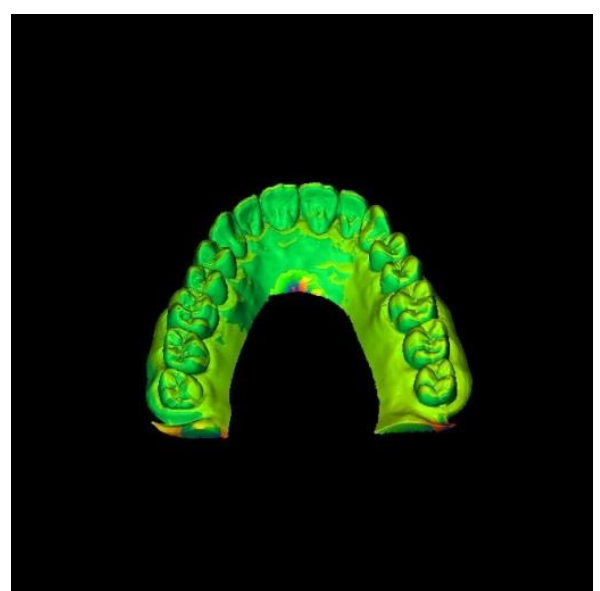

Figura 5: stl. comparativo do grupo 1 e grupo 3 com vista oclusal

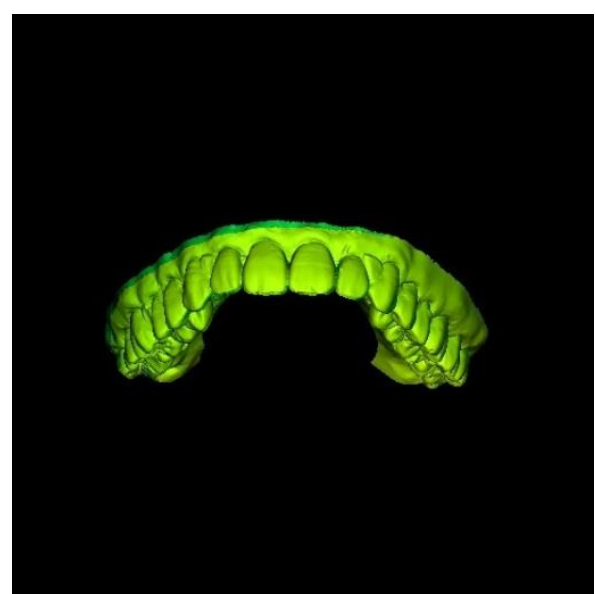

Figura 6: stl. comparativo do grupo 1 e grupo 3 com vista frontal

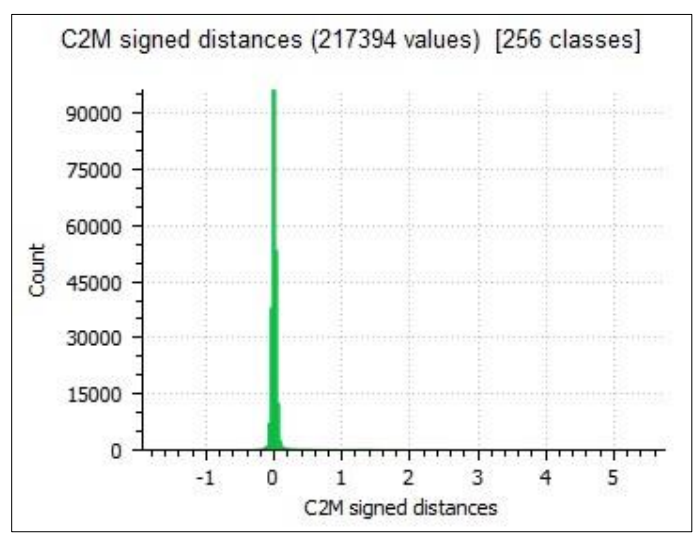

Figura 7: Variação de cor dos grupos 1 e 4 em relação as suas superficies totais comparadas e as distâncias das malhas dos stl. 


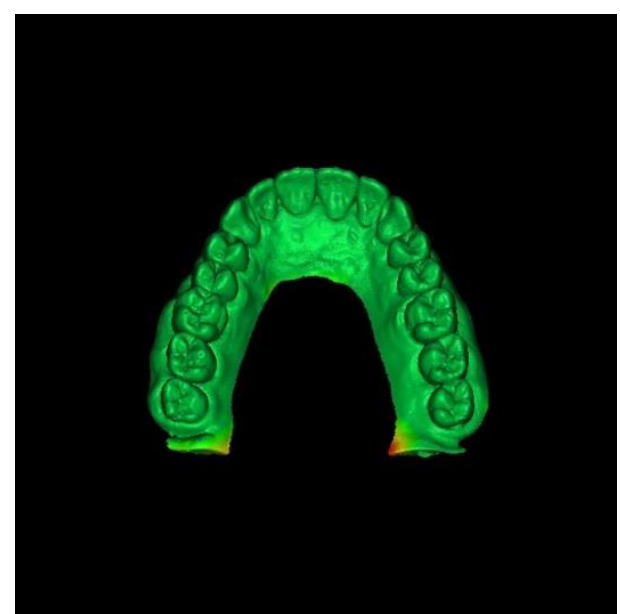

Figura 8: stl. comparativo do grupo 1 e grupo 4 com vista oclusal

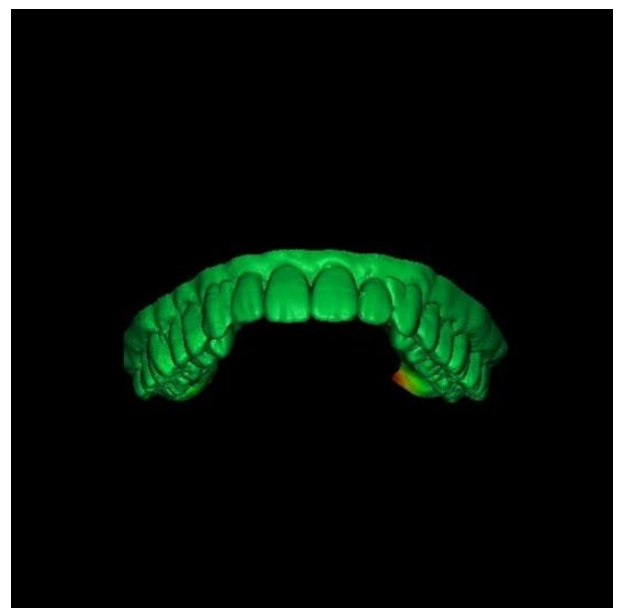

Figura 9: stl. comparativo do grupo 1 e grupo 4 com vista frontal

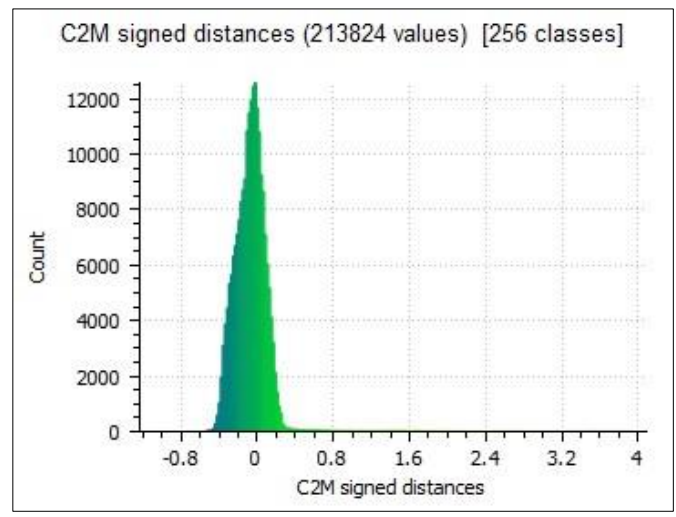

Figura 10: Variação de cor dos grupos 1 e 5.em relação as suas superficies totais comparadas e as distâncias das malhas dos stl.

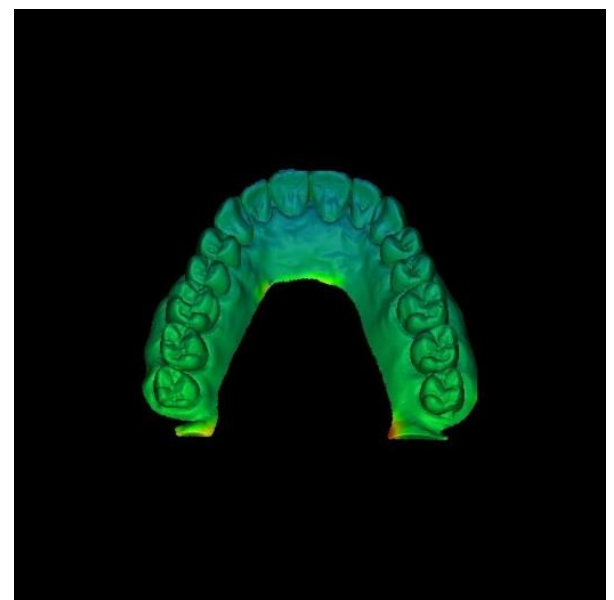

Figura 11: stl. comparativo do grupo 1 e grupo 5 com vista oclusal

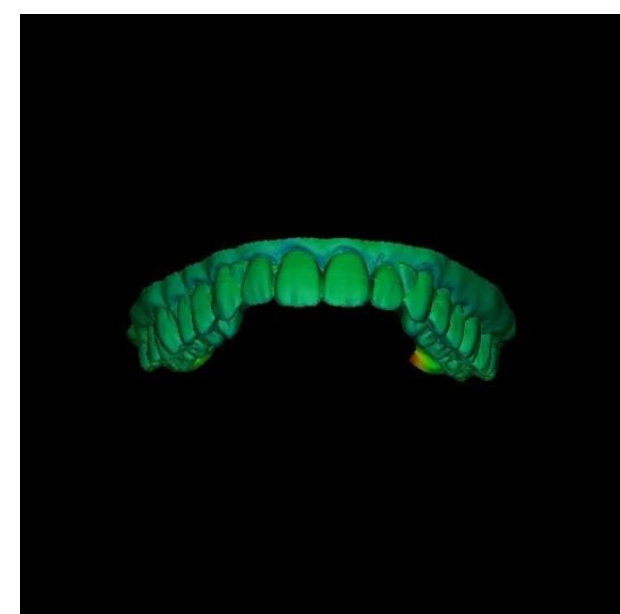

Figura 12: stl. comparativo do grupo 1 e grupo 5 com vista frontal.

$\mathrm{Na}$ quinta comparação, os grupos da mesma marca de scanner (Figura 13) foram avaliados, Grupo 2 e Grupo 3, onde é perceptível bastante alteração dimensional com maiores distorções nas regiões de vestibular e palatina dos dentes anteriores e região de terceiro molar (Figura 14 e 15). Nessa análise a quantidade de pontos captados foi de 129420.

$\mathrm{Na}$ sexta comparação (Figura 16) o Grupo 4 e Grupo 5 mostraram resultados de bastante alteração dimensional principalmente nas regiões de terceiros molares, região cervical e palatina dos dentes anteriores e nas regiões interproximais (Figura 17 e 18). Na última análise a quantidade foi de 157929 pontos

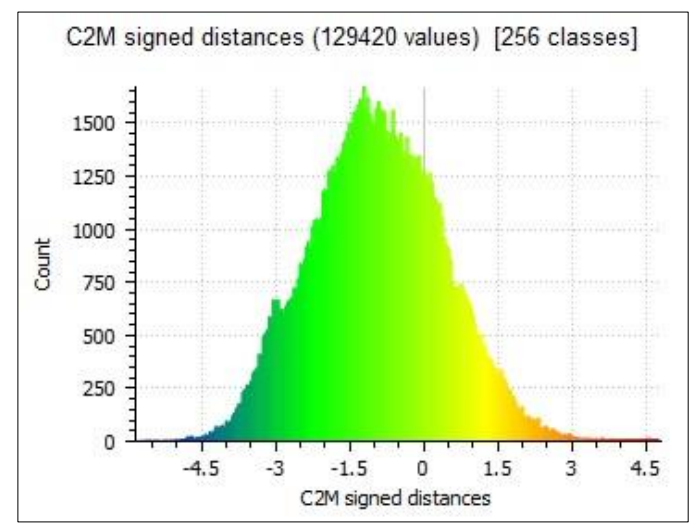

Figura 13: Variação de cor dos grupos 2 e 3 em relação as suas superficies totais comparadas e as distâncias das malhas dos stl.

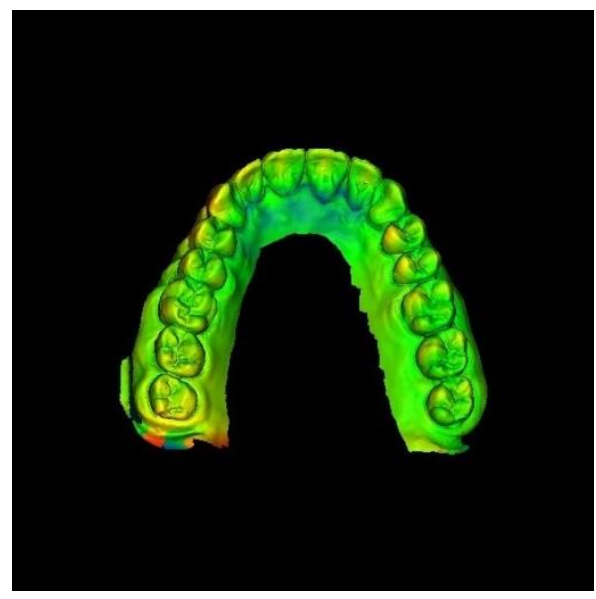

Figura 14: stl. comparativo do grupo 2 e grupo 3 com vista oclusal 


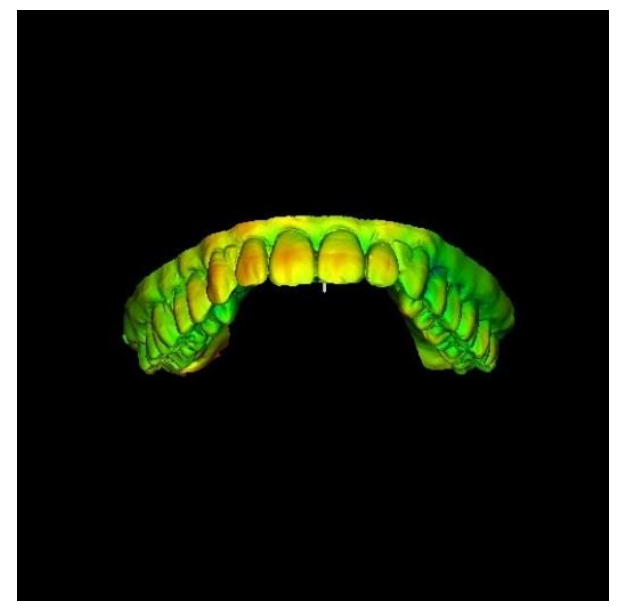

Figura 15: stl. comparativo do grupo 2 e grupo 3 com vista frontal

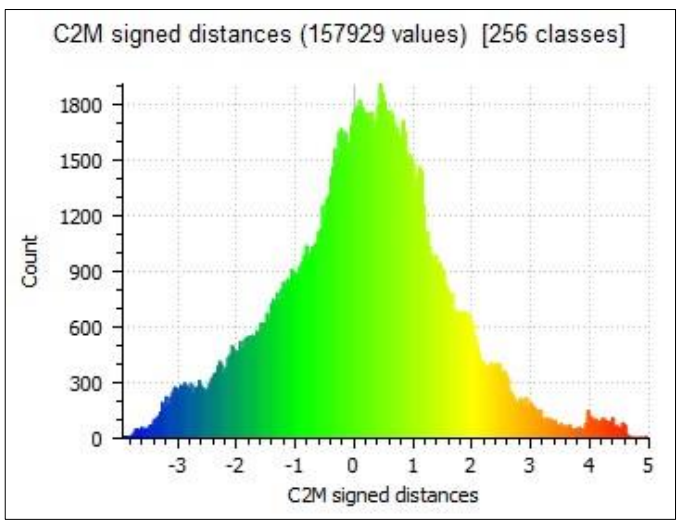

Figura 16: Variação de cor dos grupos 4 e 5 em relação as suas superficies totais comparadas e as distâncias das malhas dos stl.

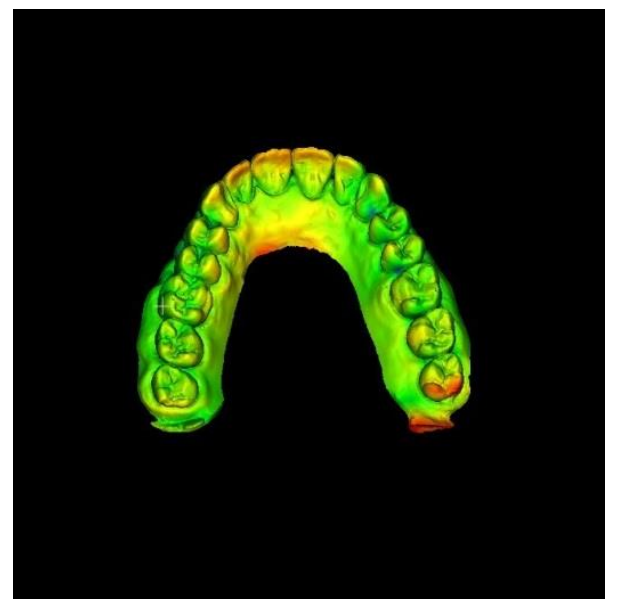

Figura 17: stl. comparativo do grupo 4 e grupo 5 com vista oclusal

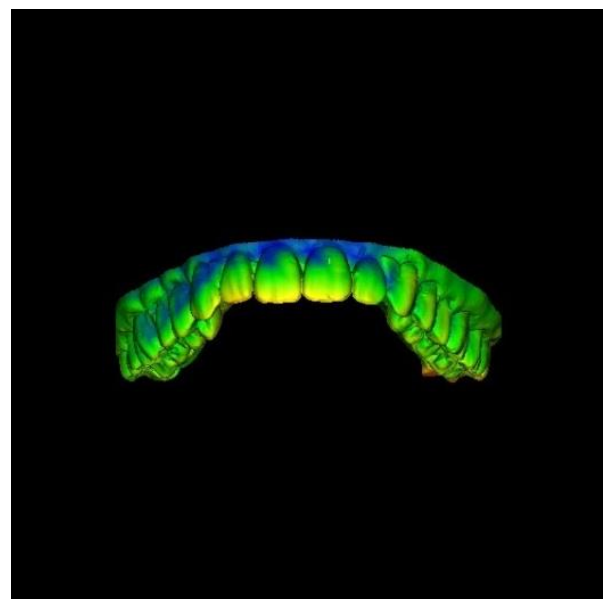

Figura 18: stl. comparativo do grupo 4 e grupo 5 com vista frontal
DISCUSSÃO

O padrão ouro de moldagens hoje em dia é o uso de materiais elastoméricos que apresentam desvantagens, quando comparados aos escaneamentos intraorais, como necessidade de desinfecção dos moldes, técnica dependente do operador, distorções inerentes à moldagem, custos e tempo de execução, entre outros ${ }^{4,5}$. A técnica de escaneamento digital intraoral tem sido relatada como uma técnica mais rápida e conveniente pela perspectiva de ambos, paciente e cirurgiãodentista $^{2-6}$. As moldagens digitais são tão precisas ou superior as moldagens tradicionais com elastômeros de acordo com a literatura ${ }^{7-8}$.

Quando utilizada a técnica intraoral realizada diretamente na cavidade oral permite um menor tempo para aquisição do modelo, porém possui algumas limitações da técnica ${ }^{9-11}$. A saliva e o movimento dos tecidos moles, associados à presença de restaurações metálicas e reflexivas, podem levar à alteração do padrão de varredura para tentar capturar superfícies perdidas ${ }^{12}$. Essas dificuldades mencionadas podem ser minimizadas fazendo a pulverização de pó na superfície, entretanto, essa camada aplicada, conforme a espessura, pode alterar o contorno do dente ${ }^{13,14}$

$\mathrm{Na}$ técnica extraoral é necessária uma moldagem convencional prévia e o modelo proveniente é escaneado com um scanner de mesa. Nessa técnica podem-se ter problemas com a confecção da moldagem e gesso o que pode alterar o resultado final. As limitações de cada técnica são inevitáveis, entretanto ambas as formas de escaneamento são reprodutíveis, válidas e confiáveis ${ }^{4,5}$

Segundo Silva ${ }^{15}$, os scanners possuem diferentes formas de captura de dados sendo a triangulação ativa, captura através de feixes de luz, utilizada pelo sistema TRIOS e a imagem confocal na qual é baseada em laser vermelho para confecção da varredura e é utilizado pelo sistema da iTero.

Em relação a estratégia de digitalização de cada scanner, embora os fabricantes recomendam um padrão específico de varredura, os clínicos passam por uma curva de aprendizado com determinado aparelho ganhando experiência de acordo com cada caso e situação clínica aparente ${ }^{16}$. Contudo diferentes padrões de varredura podem ser criados com diferentes estratégias de varredura. Esse estudo objetivou avaliar se essa mudança promove alteração dimensional e obteve como resultado que as técnicas quando modificadas pelo operador podem apresentar algum nível de 
alteração dimensional podendo comprometer a confiabilidade dos scanners.

No estudo de Muller et al. $^{16}$ foi comparada três diferentes estratégias de varredura para um único tipo de scanner TRIOS. O estudo avaliou a precisão das estratégias de varredura e se existe diferença significativa entre as técnicas, tendo como conclusão que a alteração dos padrões de digitalização influencia na precisão dos escaneamentos, corroborando com a pesquisa realizada nesse estudo, que apresenta resultados de alteração dimensional quando as técnicas são alteradas pelo operador.

Mennito et al. $^{12}$ verificaram diferentes scanners e suas respectivas estratégias de varredura recomendadas pelos fabricantes. Foi avaliado a acuracidade dos modelos digitais e comparada as técnicas de varredura dos diferentes scanners, sendo a conclusão que o padrão de varredura não afeta significativamente a precisão dos modelos digitais em sextante. Nesse estudo quando comparado 2 scanners entre si de diferentes marcas e formas de aquisição (scanner extraoral e intraoral) apresentou nível de nenhuma alteração dimensional entre as técnicas recomendadas pelo fabricante $e$, moderada alteração dimensional quando comparado a técnicas modificadas, também de diferentes formas de aquisição.

Um escaneamento fornece pontos, na qual a ligação de três pontos fornece um triangulo geométrico que tem como objetivo representar a superfície escaneada em três dimensões. A maior quantidade de pontos gera um aumento no número de dados coletados e assim gerando um menor erro e maior qualidade da imagem ${ }^{17}$. Esse estudo mostrou que os escaneamentos depois de comparados e sobrepostos, apresentou um número maior de malhas captadas pelos escaneamentos com 0 scanner de mesa quando comparado as comparações de técnicas dos scanners intraorais.

Com a crescente era da Odontologia digital se tornando comumente encontrada nas clínicas odontológicas e protéticas devemos salientar a importância dos estudos relacionados a essa tecnologia. A avaliação e comprovação da eficácia do uso desses sistemas promovem maior segurança ao cirurgião dentista frente a todas as opções de uso dos equipamentos, desde a utilização para diagnóstico, como para planejamento e tratamento de casos simples a complexos. Esse sistema permite escaneamentos eficientes porém seguindo as técnicas recomendadas pelos fabricantes que apresentam menor nível de alteração dimensional minimizando a margem de erro.

CONCLUSÃO

Houve uma alteração dimensional entre as técnicas de varredura portanto conclui-se que devemos seguir a técnica recomendada pelo fabricante na qual possui menor alteração do padrão final do modelo digitalizado principalmente entre os scanners intraorais, sendo que houve pouca alteração quando as técnicas foram comparadas com um scanner de bancada. A qualidade dos escaneamentos se baseia na quantidade de pontos coletados apresentando-se maior nos escaneamentos com scanner de mesa.

\section{REFERÊNCIAS}

1. Polido WD. Moldagens digitais e manuseio de modelos digitais: o futuro da Odontologia. Rev Dental Press J Orthod. 2010;15(5):18-22.

2. Bentz RM, Balshi SF. Complete oral rehabilitation with implants using CAD/CAM technology, stereolithography, and conoscopic holography. Rev Implant Dentistry. 2012; 21(1):8-12.

3. Kim RJY, Park JM, Shim JS. Accuracy of 9 intraoral scanners for complete-arch image aquisition: $A$ qualitative and quantitative evaluation. Rev J Prosthet Dent. 2018;120(6): 895-903.

4. Patzelt SBM, Vonau S, Stampf S, Att W. Assessing the feasibility and accuracy of digitizing edentulous jaws. JADA. 2013;144(8): 914-20.

5. Sason GK, Mistry G, Tabassum R, Shetty O. A comparative evaluation of intraoral and extraoral digital impressions: An in vivo study. Rev J Indian Prosthodont Soc. 2018;18:108-16.

6. Gjelvold B, Chrcanovic BR, Korduner EK, Collin-bagewitz I, Kisch J. Intraoral digital impression technique comparated to conventional impression technique. A randomized clinical trial. Rev J Prosthodont. 2016;25:282-87.

7. Birnbaum N, Aaronson HB, Stevens C, Cohen B. 3D digital scanners: a high-tech approach to more accurate dental impressions. Inside Dentistry. 2009:5(4).

8. Güth JF, Keul C, Stimmelmayr M, Beuer F, Edelhoff D. Accuracy of digital models obtained by direct and indirect data capturing. Clin Oral Investig. 2013;17(4):1201-208.

9. Flugge TV, Schlager S, Nelson K, Nahles $S$, Metzger MC. Precision of intraoral digital dental impressions with iTero and extraoral digitization with the iTero and a model scanner. Rev Am J Orthod Dentofacial Orthop. 2013;144(3):471-8.

10. Aragón M, Pontes L, Bichara L, Flores-Mir C, Normando D. Validity and reliability of intraoral 
scanners compared to conventional gypsum models measurements: a systematic review. Europ J Orthod. 2016;1-6.

11. Bósio AJ, Santo M, Jacob HB. Odontologia digital contemporânea - scanners intraorais digitais. Rev Orthod Sci Pract. 2017;10(39): 355-62.

12. Mennito AS, Evans ZP, Lauer AW, Patel RB, Ludlow ME, Renne WG. Evaluation of the effect scan pattern has on the trueness and precision of six intraoral digital impression systems. Rev J Esthet Restor Dent. 2018;30:113-18.

13. Ting-Shu S, Jian DS. Intraoral Digital Impression Technique: a Review. J Prosthod. 2015;24(4):313-21.

14. Feitosa EF, Emília L, Oliveira A, Chagas RB. Escaneamento Intraoral em Reabilitações Orais Protéticas. XIV Semana Acadêmica FAMETRO; 2018; Fortaleza, Brasil].

15. Silva LRR. Sistemas de moldagem digital em Odontologia [monografia]. Porto Velho: Faculdade São Lucas; 2014.

16. Müller $P$, Ender $A$, Joda $T$, Katsoulis J. Impact of digital intraoral scan strategies on the impression accuracy using the TRIOS Pod scanner. Quintessence Int. 2016;47(4):343-49.

17. Freitas JPO, Hernandez BA, Albarracín ML, Sousa EAC. Engenharia reversa na obtenção, processamento e reconstrução de imagens para remodelagem tridimensional aplicada ao cálculo em elementos finitos. VIII Congresso Nacional de Engenharia Mecânica; 2014 agosto 10-15; Uberlândia, Minas Gerais, Brasil.

\section{CONFLITO DE INTERESSES}

Os autores declaram não haver conflitos de interesse

\section{AUTOR PARA CORRESPONDÊNCIA}

\section{Maurício André Bisi}

Curso de Odontologia, FSG Centro Universitário Rua Os Dezoito do Forte, 2366. Bairro São Pelegrino. 95020-472 Caxias do Sul - RS, Brasil

Telefone: (54) 21016026/ (54) 999994949.

E-mail: maubisi@hotmail.com 